\title{
Panorama das internações por condições sensíveis à atenção primária no Espírito Santo, Brasil, 2000 a 2014
}

\author{
Overview of hospitalizations for ambulatory care sensitive conditions in Espirito Santo, Brazil, \\ 2000 to 2014 \\ Panorama de las hospitalizaciones por condiciones sensibles a la atención primaria en Espírito
} Santo, Brasil, 2000 a 2014

Rosalva Grobério Pazóa, Diana de Oliveira Frauches ${ }^{b}$, Maria del Carmen Bisi Molinaa, Nágela Valadão Cade ${ }^{a}$

\section{Resumo}

Objetivo: Descrever a série temporal das internações por condições sensiveis à atenção primária (ICSAP) no estado do Espírito Santo, no período de 2000 a 2014, segundo sexo, faixa etária, porte municipal, macrorregiões e grupos de causa, e investigar os fatores associados à ocorrência dessas hospitalizações. Métodos: Estudo ecológico de série temporal das ICSAP ocorridas de 2000 a 2014. A análise da série temporal foi realizada pelo método Cochrane-Orcutt, e para a análise de regressão multivariável multinível usou-se o modelo de regressão binomial negativa. Resultados: As ICSAP diminuíram $28,79 \%$ no período. As maiores taxas foram observadas entre os idosos e as crianças menores de cinco anos, e reduziram mais entre idosos e adultos. Os principais grupos de causas foram as gastroenterites infecciosas e complicações, a infecção no rim e trato urinário, e a insuficiência cardíaca. Houve redução das taxas de ICSAP na medida em que cresceu a taxa de cobertura da estratégia saúde da família $(0,60$, IC: $0,56-0,66)$, a proporção de médicos $(0,90$, IC: $0,84-0,96)$, de pretos e pardos $(0,88$, IC: $0,83-0,93)$ e de pessoas com ensino médio $(0,87$, IC: 0,76-0,99), enquanto houve acréscimo dessas hospitalizações a cada aumento do produto interno bruto per capita, do índice de Gini, da taxa de urbanização, dos leitos hospitalares e dos planos de saúde. Conclusão: A expansão e consolidação da estratégia saúde da família são importantes para a redução das ICSAP no estado dentre outros fatores, como recursos de saúde e fatores socioeconômicos.
Palavras-chave: Atenção Primária à Saúde Estratégia Saúde da Família Indicadores de Qualidade em Assistência à Saúde Hospitalização

\begin{abstract}
Objective: To describe the time series of hospitalizations due to ambulatory care sensitive conditions (ACSC) in Espirito Santo, from 2000 to 2014, according to sex, age, municipal size, regions and cause groups, and investigate the factors associated with the occurrence of these hospitalizations. Method: Ecological study of time series of ACSC hospitalizations occurred from 2000 to 2014 . The analysis of the time series was performed by the Cochrane-Orcutt method, and for the multivariable regression analysis the negative binomial regression model was used. Results: ACSC hospitalizations decreased $28.79 \%$ in the period. The highest rates were observed among the elderly and children under five, and decreased more among the elderly and adults. The main groups of causes were gastroenteritis and complications, infection in the kidney and urinary tract, and heart failure. There was a reduction in the ACSC hospitalizations rates as the coverage rate of family health strategy increased $(0.60, \mathrm{Cl}: 0.56-0.66)$, proportion of physicians $(0.90, \mathrm{Cl}: 0.84-0,96)$, blacks (0.88, Cl: $0.83-0.93)$ and people with high school education $(0.87, \mathrm{Cl}: 0.76-0.99)$, while there was an increase of these hospitalizations with each increase in gross domestic product per capita, Gini index, urbanization rate, hospital beds and health insurance. Conclusion: The expansion and consolidation of family health strategy are important for the reduction of ACSC hospitalizations in the state among other factors, such as health resources and socioeconomic factors.
\end{abstract}

Como citar: Pazó RG, Frauches DO, MCB Molina, Cade NV. Panorama das internações por condições sensíveis à atenção primária no Espírito Santo, Brasil, 2000 a 2014. Rev Bras Med Fam Comunidade. 2017;12(39):1-12. http://dx.doi.org/10.5712/rbmfc12(39)1546
Keywords:

Primary Health Care Family Health Strategy Quality Indicators, Health Care Hospitalization

Fonte de financiamento: declaram não haver. Parecer CEP: não se aplica. Conflito de interesses: declaram não haver. Procedência e revisão por pares: revisado por pares. Recebido em: 07/06/2017. Aprovado em: 03/07/2017. 


\section{Resumen}

Objetivo: Describir la serie temporal de las tasas de hospitalizaciones por condiciones sensibles a la atención primaria (ACSC) en Espírito Santo, de 2000 a 2014, según sexo, edad, regiones y causa, e investigar factores asociados. Métodos: Estudio ecológico de serie temporal de las hospitalizaciones por ACSC, de 2000 a 2014. El análisis de la serie temporal fue realizado por el método Cochrane-Orcutt, y para el análisis de regresión multivariable multinivel se usó el modelo de regresión binomial negativa. Resultados: Hubo reducción del $28,79 \%$. Las mayores tasas fueron observadas entre los ancianos y los niños menores de cinco años, y redujeron más entre ancianos y adultos. Las principales causas fueron las gastroenteritis infecciosas y complicaciones, la infección en el riñón y del tracto urinario, y la insuficiencia cardiaca. Se observó una reducción de las hospitalizaciones por ACSC en la medida en que creció la tasa de cobertura de la estrategia de salud de la familia $(0,60$, IC: $0,56-0,66)$, proporción de médicos $(0,90$, IC: $0,84-0,96)$, negros y pardos $(0,88$, IC: $0,83-0,93)$ y personas con escuela secundaria $(0,87, I C: 0,76-0,99)$, mientras hubo aumento de esas hospitalizaciones a cada aumento del producto interno bruto per cápita, índice de Gini, tasa de urbanización, camas hospitalarias y cobertura del plan de salud. Conclusión: La expansión y consolidación de la estrategia de salud de la familia son importantes para la reducción de las hospitalizaciones por ACSC en el estado entre otros factores, como recursos de salud y factores socioeconómicos.
Palabras clave:

Atención Primaria de Salud Estrategia de Salud Familiar Indicadores de Calidad de la Atención de Salud Hospitalización

\section{Introdução}

As internações por condições sensíveis à atenção primária (ICSAP) se tornaram internacionalmente estabelecidas como um indicador de qualidade e parte do pressuposto de que o acesso adequado ao atendimento ambulatorial efetivo pode prevenir a necessidade de hospitalização por alguns agravos. ${ }^{1-3}$

Para um atendimento oportuno, faz-se necessário o estabelecimento e manutenção de um sistema de Atenção Primária à Saúde (APS) acessível, bem distribuído, com capacidade de gerir os problemas da comunidade, com equipes de trabalho suficientes, e capacidade de realizar o manejo clínico resolutivo.

No Brasil, entre 1997 e 2007, as ICSAP reduziram 24\% em associação com o aumento progressivo de cobertura da Estratégia Saúde da Família (ESF), que tem a proposta de trabalhar com equipes de atenção à família e busca ativa de populações de risco. ${ }^{4}$ Outros estudos também descreveram a ocorrência destas hospitalizações, mostrando a utilidade do indicador na avaliação das estratégias desenvolvidas para melhorar o acesso aos serviços de saúde. ${ }^{4-7}$

No estado do Espírito Santo (ES), em meados de 1998 foi implantada a primeira equipe, em 2000 esse modelo assistencial estava presente em metade dos municípios, e a partir daí houve avanço progressivo na cobertura da ESF no estado. ${ }^{8}$ Houve redução das ICSAP no ES,1998 a 2009, com variação média anual de $5,2 \%$ entre homens e $4,7 \%$ entre mulheres, ${ }^{5}$ e essas hospitalizações representaram $22,41 \%$ das internações do estado em 2010.9

Alguns autores têm demonstrado que, além da cobertura da ESF, a melhoria das condições socioeconômicas colaboram para a redução das ICSAP. ${ }^{1,9-11}$ Nessa perspectiva, a hipótese do presente estudo consiste em que a implantação da ESF e as melhorias socioeconômicas ocorridas no ES podem ter contribuído para a redução das ICSAP em nível municipal. Busca-se, portanto, ampliar a informação sobre as ICSAP nesses municípios em período de 15 anos de observação e sua relação com a ESF, com vistas a subsidiar informações sobre as demandas da APS para determinados grupos mais afetados por essas internações, bem como orientar estratégias de treinamentos na prevenção de agravos específicos.

Objetiva-se neste estudo descrever a série temporal das ICSAP no ES, no período de 2000 a 2014 segundo sexo, faixa etária, porte municipal, macrorregiões e grupos de causa, e investigar os fatores associados à ocorrência dessas hospitalizações, tais como a disponibilidade de recursos de saúde e os determinantes socioeconômicos. 


\section{Métodos}

Estudo ecológico de séries temporais das ICSAP ocorridas nos 78 municípios do ES, no período de $1^{\circ}$ de janeiro de 2000 a 31 de dezembro de 2014. A escolha do período considerou a consolidação da ESF no estado, pois sua implantação nos municípios se deu de forma distinta e incipiente em alguns deles antes do ano 2000.

As informações sobre as internações foram extraídas do banco do Sistema de Informações Hospitalares (SIH) do Departamento de Informação e Informática do SUS (DATASUS). Analisou-se o ano de ocorrência da internação na Autorização de Internação Hospitalar (AIH) paga para definir a temporalidade do evento. Foram excluídos os partos por representarem um desfecho natural da gestação.

As taxas de ICSAP compuseram a variável resposta e foram calculadas por 10.000 habitantes. A população utilizada para o cálculo foi aquela referida pelos censos do Instituto Brasileiro de Geografia e Estatística (IBGE), bem como a população intercensitária. As taxas foram padronizadas pelo método direto, considerando a população do estado no censo de 2010 como padrão.

As ICSAP foram agrupadas segundo sexo, município de residência, faixas etárias e grupos de causas. O diagnóstico principal da AlH foi usado para definir a causa da internação e para classificação como ICSAP e seus grupos de causa seguiu-se a lista brasileira, publicada em 2008. As faixas etárias seguiram os 11 grupos adotados pelo banco do DATASUS: 0 a 4 anos; 5 a 9; 10 a 14; 15 a $19 ; 20$ a 29; 30 a $39 ; 40$ a $49 ; 50$ a $59 ; 60$ a $69 ; 70$ a $79 ; 80$ e mais.

Os municípios foram agrupados segundo o porte municipal até 20.000, de 20.001 até 100.000, de 100.001 a 300.000 , mais de 300.000 habitantes. Cabe registrar que não há, no estado, município com mais do que 500.000 habitantes. Também foi utilizada a classificação do Plano Diretor de Regionalização da Saúde do ES 2011, na qual os municípios são categorizados em macrorregiões Norte, Central, Metropolitana e Sul. A regionalização está orientada para promover o acesso, resolutividade e qualidade dos serviços de saúde. ${ }^{12}$

A análise da série temporal foi realizada pelo método Cochrane-Orcutt de regressão dos mínimos quadrados, que permitiu estimar os parâmetros de regressão e filtrar a autocorrelação temporal. ${ }^{13} \mathrm{O}$ nível de significância adotado foi de $5 \%$. Classificou-se a tendência da série como estabilidade $(p>0,05)$, redução ( $p<0,05$ e coeficiente da regressão negativo) ou crescimento ( $p<0,05$ e coeficiente da regressão positivo).

Realizou-se análise multivariável para averiguar a influência da ESF nessas hospitalizações. As variáveis explicativas foram proxies selecionadas dentre aquelas reconhecidas na literatura como determinantes das ICSAP, como as condições socioeconômicas, características geográficas, demográficas e organização dos serviços de saúde (rede privada e pública). 2,6,9,10

As variáveis explicativas seguiram as definições constantes em suas respectivas fontes: cobertura da ESF (Departamento de Atenção Básica, 2000 a 2014); taxa de pobreza, taxa de urbanização, proporção de pardos e negros, domicílios com esgotamento sanitário, renda mensal familiar per capita e taxa de analfabetismo (IBGE, 2000 e 2010); percentual de ensino médio (Programa das Nações Unidas para o Desenvolvimento, 2000 e 2010); Índice de Gini (IPEA, 2000 e 2010); produto interno bruto per capita (PIB per capita) (IBGE, 2000 a 2013); número de médicos e leitos hospitalares SUS (Cadastro Nacional de Estabelecimentos de Saúde, 2000, 2005 a 2014); cobertura de plano de saúde (Agência Nacional de 
Saúde Suplementar, 2000 a 2014). Todas as variáveis explicativas são contínuas e foram tratadas como variáveis de distribuição gaussiana.

Para aquelas variáveis com indisponibilidade de informação para todos os anos da série, foi feito cálculo por interpolação e extrapolação quando necessário, uma hipótese de tendência linear foi adotada para encontrar os valores intermediários entre dois pontos de dados e determinar a variação anual dos valores das variáveis.

Para a análise de regressão multivariável, usou-se o modelo de regressão binomial negativa inflacionada de zeros. Esse modelo foi selecionado porque é apropriado para dados de contagem que exibem sobre-dispersão, como foi o caso da variável internações sensíveis. Realizou-se análise multinível, incluíram-se como $1^{\circ}$ nível os municípios e como $2^{\circ}$ nível o ano de ocorrência das ICSAP. Para seleção das variáveis para o modelo final, foi usado o método backward, e as variáveis que apresentaram valor de $p>0,20$ foram excluídas. Calculou-se o risco relativo (RR) e o intervalo de confiança de 95\% (IC95\%).

Os fatores de inflação da variância (VIF) foram usados para detectar a presença de multicolinearidade, e as variáveis com comportamento colinear foram eliminadas. Também foram feitas análises de resíduos do modelo final pelo gráfico q-q plot.

Todas as análises estatísticas foram conduzidas no software R versão 3.2.2 (R CORE TEAM, 2016. Vienna, Austria; URL https://www.R-project.org/). A pesquisa utilizou informações que são de domínio público e por isso foi liberada da aprovação do Comitê de Ética em Pesquisa.

\section{Resultados}

No período de 2000 a 2014 ocorreram 2.444.174 internações nos 78 municípios do ES, cuja população passou de 3.097.232 para 3.885.049 habitantes, perfazendo uma taxa mediana de 470,39 internações por 10.000 habitantes, com estabilidade no período. As internações por ICSAP foram 640.187 e representaram $26,33 \%$ do total de internações, com uma taxa padronizada estadual que caiu de 146,35 por 10.000 habitantes em 2000 para 104,22 em 2014, correspondendo a uma redução de 28,79\%.

A curva estadual da taxa de ICSAP no primeiro quinquênio da análise (2000-2004) apresentou discreto aumento, com pico em 2003 e variação média anual de 0,50\%. No entanto, no período seguinte (2005-2009) foi possível notar importante queda na curva e variação média anual de $-5,52 \%$. No último período da série (2010-2014) manteve-se a tendência de redução, porém num patamar menor, -0,89\%. Esse mesmo padrão foi observado na curva de ICSAP segundo sexo (Gráfico 1).

As ICSAP diminuíram entre os idosos, adultos e na faixa pediátrica de 0 a 4 anos, com maior redução entre os dois primeiros. Assim como houve redução em todas as macrorregiões com exceção da Sul, enquanto na análise por porte municipal notou-se redução das ICSAP em todos os extratos de municípios, sendo maiores as quedas observadas naqueles de pequeno porte (menos de 100.000 habitantes) (Tabela 1).

As maiores taxas de ICSAP foram observadas entre os idosos e na faixa etária pediátrica de 0 a 4 anos. Entre adultos, as maiores taxas ocorreram com o aumento da idade. As menores taxas foram registradas na adolescência nas faixas de 10 a 14 anos e 15 a 19 anos. No entanto, chamou a atenção o fato de ter ocorrido crescimento das taxas para essa segunda faixa etária (Tabela 1). 


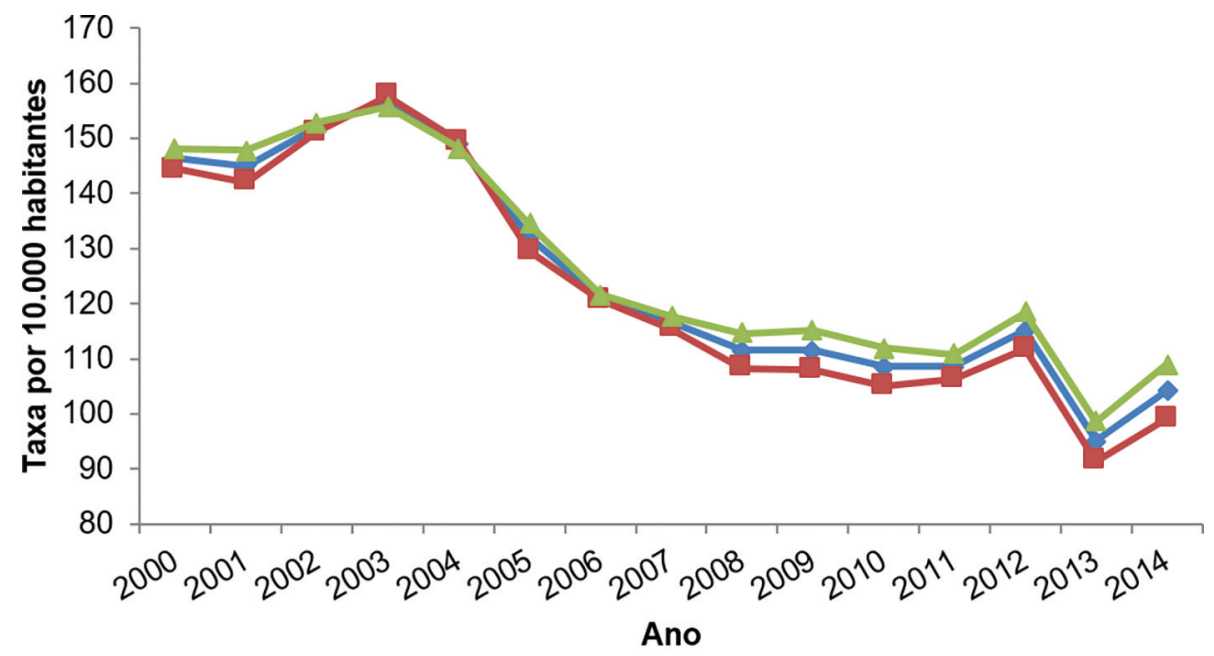

$\sim$ ICSAP total $=$ Masculino $\rightarrow$ Feminino

Gráfico 1. Série temporal das taxas de internações por condições sensíveis à atenção primária total e segundo o sexo, Espírito Santo, Brasil, 2000 a 2014. Nota: ICSAP - internações por condições sensíveis à atenção primária.

Tabela 1. Distribuição das medianas (1ำ e $3^{\circ}$ quartil) e tendência das taxas padronizadas de internação por condições sensíveis à atenção primária por 10.000 habitantes segundo sexo, faixa etária, macrorregião e porte municipal, Espírito Santo, Brasil, 2000 a 2014.

\begin{tabular}{|c|c|c|c|c|c|}
\hline Variável & Taxa de ICSAP & Modelo & $\mathbf{R}^{2}$ & Variação média anual (\%) & Tendência \\
\hline Total & $118,46(109,78-163,87)$ & $y=8749.64-4.3 x$ & 0,645 & $-3,11$ & Redução** \\
\hline \multicolumn{6}{|l|}{ Sexo } \\
\hline Feminino & $118,44(113,29-148,03)$ & $y=9337,18-4,56 x$ & 0,702 & $-1,95$ & Redução** \\
\hline \multicolumn{6}{|l|}{ Faixa etária } \\
\hline 5 a 9 anos & $66,36(61,18-69,03)$ & $y=1214,77-0,57 x$ & 0,094 & 1,27 & Estabilidade \\
\hline 10 a 14 anos & $33,25(31,67-34,94)$ & $y=-927,99+0,48 x$ & 0,232 & 1,16 & Estabilidade \\
\hline 15 a 19 anos & $39,19(37,26-43,56)$ & $y=-1912,22+0,97 x$ & 0,294 & 1,86 & Crescimento* \\
\hline 20 a 29 anos & $45,45(44,05-54,41)$ & $y=2914,34-1,43 x$ & 0,576 & $-2,62$ & Redução** \\
\hline 30 a 39 anos & $49,54(46,79-63,37)$ & $y=4419,78-2,18 x$ & 0,711 & $-4,18$ & Redução** \\
\hline 70 a 79 anos & $524,63(443,40-769,65)$ & $y=70337,62-34,74 x$ & 0,536 & $-3,81$ & Redução** \\
\hline 80 e mais & $985,43(848,25-1356,80)$ & $y=124061,45-61,24 x$ & 0,542 & $-2,79$ & Redução** \\
\hline \multicolumn{6}{|l|}{ Macrorregião } \\
\hline Metropolitana & $94,41(85,07-119,80)$ & $y=9461,01-4,66 x$ & 0,595 & $-2,60$ & Redução** \\
\hline Norte & $146,80(142,11-166,14)$ & $y=6195,28-3,01 x$ & 0,332 & $-0,37$ & Redução* \\
\hline Central & $149,63(140,51-188,96)$ & $y=12187,48-5,99 x$ & 0,684 & $-3,75$ & Redução** \\
\hline Sul & $185,91(180,92-193,00)$ & $y=2723,27-1,26 x$ & 0,237 & 0,00 & Estabilidade \\
\hline \multicolumn{6}{|l|}{ Porte } \\
\hline Até 20.000 & $157,29(145,49-188,28)$ & $y=11515,06-5,66 x$ & 0,798 & $-2,77$ & Redução** \\
\hline
\end{tabular}

ICSAP: internações por condições sensíveis à atenção primária. * $p<0,05 .{ }^{* *} p<0,01$. 
Na distribuição por macrorregiões, verificou-se maior taxa de ICSAP na Sul e a menor na Metropolitana. Os municípios de menor porte apresentaram as maiores taxas de ICSAP, enquanto as menores taxas foram observadas nos municípios com mais de 300.000 habitantes (Tabela 1).

Quanto às taxas de ICSAP segundo causas, os principais grupos foram as gastroenterites infecciosas e complicações, a infecção no rim e trato urinário, a insuficiência cardíaca, as pneumonias bacterianas e as doenças cerebrovasculares. Na análise da série temporal houve redução em oito grupos $(42,11 \%)$, estabilidade em sete $(36,84 \%)$ e aumento em quatro grupos de causa $(21,05 \%)$ (Tabela 2$)$.

Tabela 2. Distribuição das medianas ( $1^{\circ}$ e $3^{\circ}$ quartil) e tendências das taxas de internação por condições sensíveis à atenção primária por 10.000 habitantes segundo grupos de causas, Espírito Santo, Brasil, 2000 a 2014.

\begin{tabular}{|c|c|c|c|c|c|}
\hline Variável & Taxa de ICSAP & Modelo & $\mathbf{R}^{2}$ & $\begin{array}{c}\text { Variação média } \\
\text { anual (\%) }\end{array}$ & Tendência \\
\hline Gastroenterites infecciosas e complicações & $19,96(18,20-24,98)$ & $y=2487,76-1,23 x$ & 0,827 & $-2,44$ & Redução** \\
\hline Infecção no rim e trato urinário & $12,85(11,38-13,97)$ & $y=-511,07+0,26 x$ & 0,292 & 3,11 & Crescimento* \\
\hline Insuficiência cardíaca & $12,44(12,03-17,09)$ & $y=1284.4-0,63 x$ & 0,761 & $-4,92$ & Redução** \\
\hline Pneumonias bacterianas & $12,01(8,26-14,23)$ & $y=1422,95-0,7 x$ & 0,186 & $-1,23$ & Estabilidade \\
\hline Doenças cerebrovasculares & $9,20(7,29-10,04)$ & $y=-876,86+0,44 x$ & 0,098 & 0,07 & Estabilidade \\
\hline Asma & $7,69(5,78-12,75)$ & $y=1466,79-0,73 x$ & 0,406 & $-8,03$ & Redução** \\
\hline Doenças pulmonares & $7,09(5,94-8,85)$ & $y=589,12-0,29 x$ & 0,318 & $-4,21$ & Redução* \\
\hline Hipertensão & $7,03(5,77-10,66)$ & $y=1080,87-0,53 x$ & 0,559 & $-5,83$ & Redução** \\
\hline Angina & $6,09(5,58-6,41)$ & $y=-96,05+0,05 x$ & 0,035 & 5,58 & Estabilidade \\
\hline Diabetes melitus & $5,65(5,31-6,39)$ & $y=-89,56+0,05 x$ & 0,033 & 0,27 & Estabilidade \\
\hline Úlcera gastrointestinal & $4,52(1,97-6,67)$ & $y=884,72-0,44 x$ & 0,353 & $-4,98$ & Redução* \\
\hline Infecção da pele e tecido subcutâneo & $4,43(3,80-5,37)$ & $y=-549,02+0,28 x$ & 0,407 & 5,22 & Crescimento** \\
\hline Deficiências nutricionais & $3,33(2,95-3,41)$ & $y=219,99-0,11 x$ & 0,495 & $-0,48$ & Redução** \\
\hline Doença inflamatória órgãos pélvicos femininos & $2,21(1,91-2,71)$ & $y=281,46-0,14 x$ & 0,935 & $-6,30$ & Redução** \\
\hline Epilepsias & $2,07(1,83-2,34)$ & $y=49,9-0,02 x$ & 0,066 & $-0,49$ & Estabilidade \\
\hline Doenças relacionadas ao pré-natal e parto & $1,06(0,98-1,48)$ & $y=-237,79+0,12 x$ & 0,510 & 6,24 & Crescimento** \\
\hline D. prev. por imunização e condições sensíveis & $1,02(0,80-1,26)$ & $y=-13,16+0,01 x$ & 0,005 & $-1,10$ & Estabilidade \\
\hline Infecções de ouvido, nariz e garganta & $0,84(0,39-0,91)$ & $y=-246,22+0,12 x$ & 0,466 & 11,15 & Crescimento** \\
\hline Anemia & $0,74(0,60-0,79)$ & $y=46,39-0,02 x$ & 0,209 & $-0,25$ & Estabilidade \\
\hline
\end{tabular}

ICSAP: internações por condições sensíveis à atenção primária. * $p<0,05 .{ }^{* *} p<0,01$.

A proporção de cobertura da ESF cresceu $151 \%$ e, considerando os 15 anos da série, em média 47 $(60,26 \%)$ municípios ofereceram cobertura maior que $70 \%$. A macrorregião Sul apresentou a maior média de cobertura de ESF $(80,23 \%)$, seguida pela Central $(70,11 \%)$, a Norte $(63,46 \%)$ e por último a Metropolitana $(60,68 \%)$. Ainda, segundo porte municipal, as maiores coberturas médias foram vistas nos municípios com menos de 20.000 habitantes $(84,00 \%)$ e naqueles de 20.000 a 100.000 habitantes $(60,80 \%)$, enquanto nos de médio porte $(34,64 \%)$ e grande porte $(26,81)$ essa proporção caiu de forma expressiva.

Ao longo dos 15 anos houve aumento da renda média domiciliar per capita, da proporção de pessoas maiores de 18 anos com ensino médio completo, da taxa de esgotamento sanitário, queda das taxas de pobreza e das taxas de analfabetismo, indicando melhora nas condições socioeconômicas. Ainda, observou-se discreta redução do índice de Gini, apontando para uma possível redução da desigualdade social. Quanto aos recursos de saúde, houve aumento da proporção de médicos por 1.000 habitantes, da cobertura de plano de saúde, e redução da proporção de leitos hospitalares SUS (Tabela 3). 
Tabela 3. Distribuição das médias e desvio padrão das variáveis explicativas, Espírito Santo, Brasil, 2000 a 2014.

\begin{tabular}{|c|c|c|c|c|}
\hline Variáveis & 2000 & 2014 & Diferença & Variação (\%) \\
\hline Cobertura da ESF${ }^{1}(\%)$ & $34,90(42,53)$ & $87,60(21,75)$ & 52,70 & 151,00 \\
\hline Taxa de pobreza ${ }^{2}(\%)$ & $56,22(10,41)$ & $36,88(9,61)$ & $-19,38$ & $-34,40$ \\
\hline Taxa urbanização² (\%) & $56,91(22,84)$ & $64,92(20,03)$ & 8,01 & 14,07 \\
\hline Proporção de pretos e pardos ${ }^{2}(\%)$ & $45,98(14,30)$ & $52,87(14,38)$ & 6,89 & 14,98 \\
\hline Domicílios com esgotamento adequado ${ }^{2}(\%)$ & $48,05(21,80)$ & $57,94(17,46)$ & 9,89 & 20,58 \\
\hline Renda média domiciliar per capita ${ }^{2}(\mathrm{R} \$)$ & $415,35(146,13)$ & $564,48(199,34)$ & 149,13 & 35,90 \\
\hline Taxa de analfabetismo ${ }^{2}(\%)$ & $15,73(4,99)$ & $12,12(4,27)$ & $-3,61$ & $-22,95$ \\
\hline Ensino médio ${ }^{3}(\%)$ & $16,84(6,60)$ & $27,85(8,38)$ & 11,01 & 65,38 \\
\hline Índice de Gini ${ }^{4}$ & $0,56(0,05)$ & $0,50(0,04)$ & $-0,06$ & $-10,71$ \\
\hline Produto interno bruto per capita $(\mathrm{R} \$)^{5}$ & $5.366,51(3.611,39)$ & $30.418,97(83639,29)$ & $25.052,46$ & 466,83 \\
\hline Médicos por 1000 habitantes $^{6}$ & $0,43(0,78)$ & $1,04(0,83)$ & 0,61 & 141,86 \\
\hline Leitos SUS por 1000 habitantes $^{6}$ & $1,89(1,69)$ & $1,37(1,31)$ & $-0,52$ & $-27,51$ \\
\hline Cobertura plano de saúde $(\%)^{7}$ & $7,72(9,75)$ & $14,33(10,99)$ & 6,61 & 85,62 \\
\hline
\end{tabular}

${ }^{1}$ ESF: Estratégia Saúde da Família. Departamento de Atenção Básica, Ministério da Saúde, Secretaria de Atenção à Saúde, 2000 a 2014; ${ }^{2}$ IBGE, 2000 ,

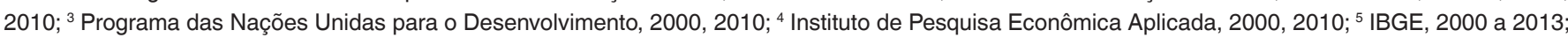
${ }^{6}$ Cadastro Nacional de Estabelecimentos de Saúde, 2000, 2005 a 2014; ${ }^{7}$ Agência Nacional de Saúde Suplementar, 2000 a 2014.

As variáveis taxa de pobreza, taxa de analfabetismo e proporção de domicílios com esgotamento sanitário foram excluídas da regressão multivariável por terem apresentado $p>0,20$, e a variável renda foi eliminada devido à multicolinearidade.

Houve redução das taxas de ICSAP na medida em que cresceu a taxa de cobertura da ESF $(0,60$, IC: 0,56-0,66), a proporção de médicos $(0,90, \mathrm{IC}: 0,84-0,96)$, de pretos e pardos $(0,88, \mathrm{IC}: 0,83-0,93)$ e de pessoas com ensino médio $(0,87$, IC: $0,76-0,99)$, enquanto ocorreu acréscimo a cada aumento do PIB per capita, $(1,04$, IC: 1,02-1,07), do índice de Gini $(1,22$, IC: 1,17-1,28), da taxa de urbanização $(1,54$, IC: 1,40-1,69), dos leitos hospitalares SUS $(1,60$, IC: 1,44-1,78) e cobertura dos planos de saúde $(1,20$ IC: 1,09-1,32) (Tabela 4).

Tabela 4. Análise multivariável das variáveis explicativas e risco de internação por condições sensíveis à atenção primária, Espírito Santo, Brasil, 2010 a 2014.

\begin{tabular}{lcc} 
Variáveis & RR (IC95\%) & p valor \\
\hline Cobertura da Estratégia Saúde da Família & $0,60(0,56-0,66)$ & 0,000 \\
Produto interno bruto per capita & $1,04(1,02-1,07)$ & 0,000 \\
Proporção de pardos e negros & $0,88(0,83-0,93)$ & 0,000 \\
Índice de Gini & $1,22(1,17-1,28)$ & 0,000 \\
Percentual de ensino médio & $0,87(0,76-0,99)$ & 0,034 \\
Taxa de urbanização & $1,54(1,40-1,69)$ & 0,000 \\
Leitos SUS por 1000 habitantes & $1,60(1,44-1,78)$ & 0,000 \\
Cobertura de plano de saúde & $1,20(1,09-1,32)$ & 0,000 \\
Médicos por 1000 habitantes & $0,90(0,84-0,96)$ & 0,003
\end{tabular}

RR: risco relativo; IC: intervalo de confiança; SUS: Sistema Único de Saúde.

\section{Discussão}

No presente estudo foi possível notar importante redução das ICSAP relacionada ao aumento da cobertura da ESF no ES de 2000 a 2014. Este decréscimo ocorreu em quase todo o estado e foi maior em municípios de pequeno porte, mas se deu de forma desigual nas diferentes faixas etárias. A maior 
proporção de médicos por habitantes diminuiu a ocorrência das ICSAP, enquanto a maior oferta de leitos favoreceu o seu crescimento. Assim como, a maior desigualdade social aumentou essas hospitalizações e o maior percentual da população com ensino médio colaborou para a sua redução.

A redução das ICSAP acompanhou a tendência descrita para o país e alguns estados, ${ }^{4-7}$ mas a curva de distribuição das ICSAP não foi uniforme ao longo do tempo, pois apresentou um aumento inicial com posterior queda. Acredita-se que possa ser reflexo do atendimento inicial pela APS a uma demanda reprimida, enquanto num segundo momento, com o aumento da cobertura da ESF, foi possível reduzir essas hospitalizações. 4,6,7,14,15 Destaca-se que o total das ICSAP no ES diminuiu, enquanto as internações como um todo, ICSAP e não ICSAP, mantiveram-se estáveis. Isso afasta a hipótese da queda detectada ser consequência de uma tendência de redução das hospitalizações em geral.

Quanto à distribuição por sexo, não se notou diferenças marcantes conforme o descrito em outros estudos que, em sua maioria, apontaram a maior ocorrência das ICSAP em homens. ${ }^{4,7,16}$

Em consonância com outros estudos, a maioria das ICSAP ocorreu entre menores de cinco anos e maiores de 60 anos. ${ }^{4,7,16}$ As altas taxas verificadas entre os idosos foram compatíveis com pesquisa semelhante realizada no Rio de Janeiro, que também percebeu redução importante na série de 2000 a $2010 .{ }^{17}$ Já as taxas encontradas para as crianças foram menores do que as vistas em estudos feitos no Piauí e Pernambuco, nos quais foi verificada redução das taxas. ${ }^{10,18} \mathrm{O}$ maior acometimento tem sido, no caso dos idosos, atribuído à maior prevalência de comorbidades e doenças crônicas, ao passo que para a faixa etária da infância a maior suscetibilidade a doenças infecciosas como gastroenterites e pneumonias são mais relatadas.

As reduções observadas entre os adultos e idosos foram maiores que nos menores de cinco anos, e chama a atenção que houve estabilidade nas faixas da infância e crescimento entre os adolescentes de 15 a 19 anos. Ao contrário, pesquisa conduzida em Minas Gerais, 1999 a 2007, observou redução dessas internações, tanto em crianças quanto em adolescentes, mas também destacou que as quedas foram menores que as notadas entre adultos. ${ }^{19}$

No que tange à análise dos grupos de causas mais frequentes, foi verificado que se compõem de doenças infecciosas e de crônicas não transmissíveis, assim como o identificado em outras pesquisas. ${ }^{4-6,15}$ Isto evidencia a forma de transição epidemiológica que ocorre no Brasil, ou seja, prolongada, com uma superposição entre as etapas e persistência de ambos os grupos de agravos, o que exige dos serviços de saúde uma abordagem abrangente em seus recursos e tecnologias para o enfrentamento desta situação. ${ }^{15,20}$

Cumpre ressaltar a redução de muitos agravos dentro do grupo das doenças crônicas, tais como a insuficiência cardíaca, asma, doenças pulmonares, hipertensão e úlcera gástrica, o que tem sido associado ao bom desempenho da ESF no manejo dessas condições, ao desenvolvimento de protocolos que colaboram para o diagnóstico e tratamento adequados e ao fornecimento de medicamentos gratuitos e apropriados. ${ }^{6,7,15}$

Por outro lado, destaca-se com preocupação o aumento e estabilidade de agravos, tais como infecção no rim e trato urinário, doenças relacionadas ao pré-natal e parto, doenças imunopreviníveis e anemia, cujo controle e tratamento concernem ações de manejo próprios da equipe de saúde da APS, porém com demandas também de intervenções de cunho sociocultural para melhorar a adesão ao acompanhamento e tratamento. $6,7,14$ 
A análise das regiões pôde identificar que a macrorregião Sul apresentou as maiores taxas de ICSAP e não acompanhou a tendência de redução das demais. Paradoxalmente, essa é a região que apresentou a maior proporção de cobertura de ESF do estado, de modo que se percebe possível influência de outros fatores tais como a grande oferta de leitos hospitalares, 2,18 leitos por 1.000 habitantes nessa região contra 1,42 da região Metropolitana.

Embora os municípios com menos do que 100.000 habitantes tenham maior cobertura da ESF, neles observou-se um maior número de internações em relação àqueles de maior porte. Estudo conduzido no Rio Grande do Sul sugeriu que apesar do progresso da APS, a existência de leitos hospitalares ociosos induz a sua utilização ainda que seja com internações evitáveis, enquanto nos municípios de grande porte a ocupação decorre das demandas de alta complexidade. ${ }^{21}$ Ainda, a proporção de médicos nos municípios de pequeno porte é $49 \%$ menor que naqueles de médio porte e $70 \%$ menor em relação aos de grande porte, ${ }^{22}$ o que traz a questão de que podem ocorrer falhas nos serviços da ESF por falta de médicos.

Na análise multivariável pôde-se averiguar que o aumento da cobertura da ESF reduz as ICSAP, e com isso diminui a carga de adoecimento e os custos financeiros, e vários são os mecanismos explicativos, como a melhora do acesso a diagnóstico e tratamento precoce aos agravos, tanto agudos como crônicos. $5,7,10,15$ Também contribui a ampliação dos recursos assistenciais e o investimento em diretrizes para abordagem de diversos agravos, dentre eles, hipertensão arterial, dengue, tuberculose e hanseníase. Outro mecanismo segundo autores é que o acompanhamento familiar proporcionado pela ESF tem mostrado associação à redução de agravos crônicos, ocasionando menos internações por essas doenças. ${ }^{7}$

A maior proporção de médicos está associada à redução das ICSAP e espera-se que isso seja consequência da presença na APS. ${ }^{1,3}$ No Brasil, a partir da implantação da ESF, grandes foram os incentivos para a fixação deste profissional na atenção básica, oferta de postos de trabalho, melhoria de salário e maior número de especializações na área.

Em contrapartida, quanto maior a oferta de leitos maior a incidência de ICSAP, provavelmente em decorrência de demanda de internações induzida por essa oferta. Assim quanto maior a disponibilidade de serviços, leitos e equipamentos, maior a chance de utilização, seja pela agilidade de acesso aos recursos secundários, que criam um padrão de utilização pelos usuários, ou a facilidade dos médicos em interná-los. ${ }^{1,6,9,21}$

Quanto aos planos de saúde, sua maior cobertura esteve associada a mais hospitalizações, oposto do resultado de estudo transversal feito no ES em 2010. ${ }^{9}$ Pode ser que a série temporal de 15 anos tenha captado um efeito não verificado no estudo anterior. Estudo das microrregiões do Brasil, 1999 a 2007, encontrou que mesmo após o ajuste para a cobertura de planos de saúde, a cobertura da ESF mostrou-se uma variável relacionada à queda das ICSAP. ${ }^{6}$

A ruralidade aumenta o risco de ICSAP segundo autores que defendem que nessas regiões há menor acesso aos cuidados primários, o que aumenta a chance de internação por agravamentos das doenças. . $2,11^{1}$ Todavia, isto não se confirmou neste estudo, e postula-se que a proximidade a aglomerados urbanos tem sido associada a mais hospitalizações, talvez pela proximidade a serviços de urgência e emergência, o que facilita o acesso a internação. ${ }^{9,23}$

Sabe-se que, além dos recursos de saúde, as condições socioeconômicas influenciam as ICSAP1,2,6,9,10 e, no período estudado, pôde-se verificar importante melhoria nesse aspecto no ES. No entanto, apenas 
o aumento da proporção de pessoas com ensino médio esteve relacionado à redução das ICSAP. Os avanços na educação da população podem colaborar para melhorar o autocuidado e por essa via reduzir internações evitáveis, além do que, podem também estar associados ao progresso socioeconômico e, daí, a melhores condições de vida.

Corroborando com a constatação da influência dos fatores socioeconômicos nas ICSAP, a cada acréscimo do índice de Gini, o que evidencia aumento da desigualdade entre os municípios, foi acompanhado de aumento do risco dessas hospitalizações. Em estudo que usou o índice de desenvolvimento humano também foi percebido maior risco de ICSAP associado a piora do indicador. ${ }^{10}$

A realidade norte-americana mostra existir grande disparidade racial nas ICSAP, no sentido de que negros internam mais, o que justificam pelas piores condições socioeconômicas e consequentemente na dificuldade de acesso a serviços de promoção e prevenção de saúde. ${ }^{11,24}$ De forma oposta, neste estudo, a maior proporção de pardos e negros nos municípios esteve associada a menores taxas de ICSAP, dado esse compartilhado por outros estudos nacionais, ${ }^{9,25}$ e entende-se em uma outra vertente que, apesar de os serviços de saúde de cuidados primários no país terem proporcionado mais atendimento, ainda há um segmento da população de pouco acesso a eles e, por isso, não chegam aos locais das internações.

A relação entre aumento do PIB per capita e aumento das ICSAP está ao contrário do esperado. Destaca-se que na macrorregião Sul, onde se notou o maior número de internações, os municípios têm o PIB per capita elevado devido à arrecadação de royalties de petróleo, mas não significa que a região esteja investindo em ações de promoção e prevenção de saúde próprias da APS na região.

Limitações do estudo

Estudos ecológicos apresentam como limitação a falácia ecológica e não se pode fazer inferências em nível individual. Outra limitação consistiu no uso de interpolação dos dados, todavia, esse método foi utilizado em outros trabalhos que pesquisaram ICSAP com sucesso, e acredita-se que a tendência geral exibida por seus valores tenha permanecido. ${ }^{6,7,10} \mathrm{~A}$ análise abrangeu apenas as internações do SUS, mas há que se considerar que elas representam cerca de $70 \%$ das hospitalizações que ocorrem no país. ${ }^{26}$ Ainda, em relação ao banco pesquisado, SIH/SUS, há questionamentos sobre a qualidade as informação e isso tem sido objeto de estudos que mostram a potencialidade de seu uso em análises epidemiológicas. ${ }^{27}$

\section{Conclusão}

Esse estudo identificou a importância da expansão e consolidação da ESF na redução das ICSAP no ES, dentre outros fatores, como maior proporção de médicos e ensino médio na população, além de apontar aqueles associados a maior ocorrência da ICSAP, tais como maior disponibilidade de leitos hospitalares, urbanização e desigualdade social.

Dessa forma, contribuiu para a ampliação de conhecimentos sobre o indicador ICSAP em nosso meio e mostra como este pode ser útil no diagnóstico situacional local subsidiando programação de ações e a correta aplicação de recursos de saúde. 


\section{Referências}

1. Burgdorf F, Sundmacher L. Potentially avoidable hospital admissions in Germany: an analysis of factors influencing rates of ambulatory care sensitive hospitalizations. Dtsch Arztebl Int. 2014;111(13):215-23. DOI: 10.3238/arztebl.2014.0215

2. Laditka JN, Laditka SB, Probst JC. Health care access in rural areas: evidence that hospitalization for ambulatory care-sensitive conditions in the United States may increase with the level of rurality. Health Place. 2009;15(3):731-40. DOI: http://dx.doi.org/10.1016/j. healthplace.2008.12.007

3. Laditka JN, Laditka SB, Probst JC. More may be better: evidence of a negative relationship between physician supply and hospitalization for ambulatory care sensitive conditions. Health Serv Res. 2005;40(4):1148-66. DOI: http://dx.doi.org/10.1111/j.1475-6773.2005.00403.x

4. Dourado I, Oliveira VB, Aquino R, Bonolo P, Lima-Costa MF, Medina MG, et al. Trends in primary health care-sensitive conditions in Brazil: the role of the Family Health Program (Project ICSAP-Brazil). Med Care. 2011;49(6):577-84. DOI: http://dx.doi.org/10.1097/ MLR.0b013e31820fc39f

5. Boing AF, Vicenzi RB, Magajewski F, Boing AC, Moretti-Pires RO, Peres KG, et al. Redução das internações por condições sensíveis à atenção primária no Brasil entre 1998-2009. Rev Saúde Pública. 2012;46(2):359-66. DOI: http://dx.doi.org/10.1590/S003489102012005000011

6. Macinko J, de Oliveira VB, Turci MA, Guanais FC, Bonolo PF, Lima-Costa MF. The influence of primary care and hospital supply on ambulatory care-sensitive hospitalizations among adults in Brazil, 1999-2007. Am J Public Health. 2011;101(10):1963-70. DOI: http:// dx.doi.org/10.2105/AJPH.2010.198887

7. Macinko J, Dourado I, Aquino R, Bonolo Pde F, Lima-Costa MF, Medina MG, et al. Major expansion of primary care in Brazil linked to decline in unnecessary hospitalization. Health Aff (Millwood). 2010;29(12):2149-60. DOI: http://dx.doi.org/10.1377/hlthaff.2010.0251

8. Brasil. Ministério da Saúde. Secretária de Assistência à Saúde. Departamento de Atenção Básica (DAB). Teto, credenciamento e implantação das estratégias de Agentes Comunitários de Saúde, Saúde da Família e Saúde Bucal. Brasília: Ministério da Saúde. [acesso 2016 Jan 15]. Disponível em: http://dab.saude.gov.br/portaldab/historico_cobertura_sf.php

9. Pazo RG, Frauches DO, Molina MCB, Cade NV. Modelagem hierárquica de determinantes associados a internações por condições sensíveis à atenção primária no Espírito Santo, Brasil. Cad Saúde Pública. 2014;30(9):1891-902. DOI: http://dx.doi.org/10.1590/0102311X00099913

10. Carvalho SC, Mota E, Dourado I, Aquino R, Teles C, Medina MG. Hospitalizations of children due to primary health care sensitive conditions in Pernambuco State, Northeast Brazil. Cad Saúde Pública. 2015;31(4):744-54. DOI: http://dx.doi.org/10.1590/0102-311X00069014

11. Hakeem FB, Howard DL, Carey TS, Taylor YJ. Differential Effects of Race and Poverty on Ambulatory Care Sensitive Conditions. J Health Dispar Res Pract. 2009;3(1):83-98.

12. Plano Diretor de Regionalização da Saúde - PDR. Secretaria de Estado da Saúde do Espírito Santo. Espírito Santo; 2011. [acesso 2015 Fev 10].Disponível em: http://saude.es.gov.br/Media/sesa/SISPACTO/PDR-Plano\%20Diretor\%20de\%20Regionaliza\%C3\%A7\%C3\%A3o_ ES_2011.pdf

13. Antunes JL, Waldman EA. Trends and spatial distribution of deaths of children aged 12-60 months in São Paulo, Brazil, 1980-98. Bull World Health Organ. 2002;80(5):391-8.

14. Alfradique ME, Bonolo PF, Dourado I, Lima-Costa MF, Macinko J, Mendonça CS, et al. Internações por condições sensíveis à atenção primária: a construção da lista brasileira como ferramenta para medir o desempenho do sistema de saúde (Projeto ICSAP - Brasil). Cad Saúde Pública. 2009;25(6):1337-49. DOI: http://dx.doi.org/10.1590/S0102-311X2009000600016

15. Brasil VP, Costa JSD. Hospitalizações por condições sensíveis à atenção primária em Florianópolis, Santa Catarina - estudo ecológico de 2001 a 2011. Epidemiol Serv Saúde. 2016;25(1):75-84. DOI: http://dx.doi.org/10.5123/S1679-49742016000100008

16. Botelho JF, Portela MC. Risco de interpretação falaciosa das internações por condições sensíveis à atenção primária em contextos locais, Itaboraí, Rio de Janeiro, Brasil, 2006-2011. Cad Saúde Pública. 2017;33(3):e00050915. DOI: http://dx.doi.org/10.1590/0102$311 \times 00050915$

17. Marques AP, Montilla DER, Almeida WS, Andrade CLT. Hospitalization of older adults due to ambulatory care sensitive conditions. Rev Saúde Pública. 2014;48(5):817-26. DOI: http://dx.doi.org/10.1590/S0034-8910.2014048005133 
18. Barreto JOM, Nery IS, Costa MSC. Estratégia Saúde da Família e internações hospitalares em menores de 5 anos no Piauí, Brasil. Cad Saúde Pública. 2012;28(3):515-26. DOI: http://dx.doi.org/10.1590/S0102-311X2012000300012

19. Santos LA, Oliveira VB, Caldeira AP. Internações por condições sensíveis à atenção primária entre crianças e adolescentes em Minas Gerais, 1999-2007. Rev Bras Saude Mater Infant. 2016;16(2):169-78. DOI: http://dx.doi.org/10.1590/1806-93042016000200006

20.Schramm JMA, Oliveira AF, Leite IC, Valente JG, Gadelha AMJ, Portela MC, et al. Transição epidemiológica e o estudo de carga de doença no Brasil. Ciênc Saúde Coletiva. 2004;9(4):897-908. DOI: http://dx.doi.org/10.1590/S1413-81232004000400011

21. Souza LL, Costa JSD. Internações por condições sensíveis à atenção primária nas coordenadorias de saúde no RS. Rev Saúde Pública. 2011;45(4):765-72. DOI: http://dx.doi.org/10.1590/S0034-89102011000400017

22. Brasil. Ministério da Saúde. Cadastro Nacional dos Estabelecimentos de Saúde do Brasil. [acesso 2016 Jan 15]. Disponível em: http: tabnet.datasus.gov.br/cgi/deftohtm.exe?cnes/cnv/profides.def

23. Batista SRR, Jardim PCBV, Sousa ALL, Salgado CM. Hospitalizações por condições cardiovasculares sensíveis à atenção primária em municípios goianos. Rev Saúde Pública. 2012;46(1):34-42. DOI: http://dx.doi.org/10.1590/S0034-89102012005000001

24. Will JC, Nwaise IA, Schieb L, Zhong Y. Geographic and Racial Patterns of Preventable Hospitalizations for Hypertension: Medicare Beneficiaries, 2004-2009. Public Health Reports. 2014;129(1):8-18. [acesso 2016 Jan 10]. PMCID:PMC3862999 Disponível em: https:// www.ncbi.nlm.nih.gov/pmc/articles/PMC3862999/

25. Oliveira AC. Ensaios sobre atenção pública à saúde em Minas Gerais [Dissertação de mestrdo]. Belo Horizonte: Universidade Federal de Minas Gerais; 2007. [acesso 2012 Jan 10]. Disponível em: http://www.bibliotecadigital.ufmg.br/dspace/handle/1843/AMSA-7EKJRW

26. Carvalho DMT. Financiamento da assistência médico-hospitalar no Brasil. Ciênc Saúde Coletiva. 2007;12(4):879-92. DOI: http://dx.doi. org/10.1590/S1413-81232007000400010

27. Lima CRA, Schramm JMA, Coeli CM, Silva MEM. Revisão das dimensões de qualidade dos dados e métodos aplicados na avaliação dos sistemas de informação em saúde. Cad Saúde Pública. 2009;25(10):2095-109. DOI: http://dx.doi.org/10.1590/S0102-311X2009001000002

a Programa de Pós-graduação em Saúde Coletiva, Universidade Federal do Espírito Santo (UFES). Vitória, ES, Brasil. rosalvapazo@gmail.com (Autora correspondente); mdmolina@uol.com.br; nagelavc@gmail.com

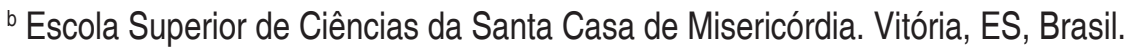
diana.frauches@emescam.br 